
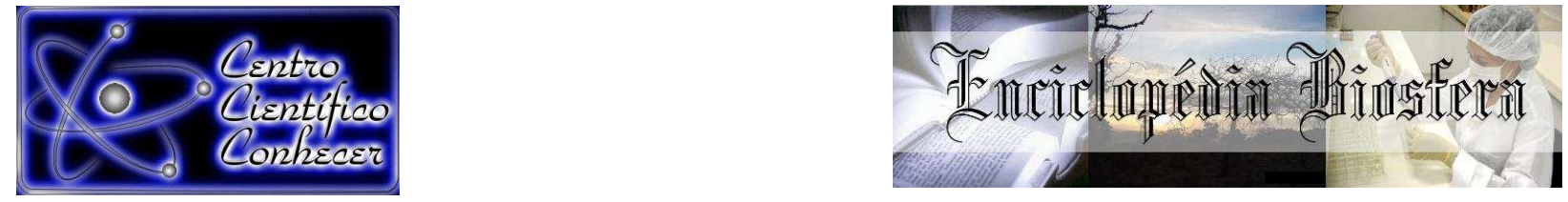

\title{
TESTE DE ALGORITMOS DE CLASSIFICAÇÃO PARA A ANÁLISE TEMPORAL DE USO E OCUPAÇÃO DO SOLO NO MƯNICÍPIO DE SÃO GABRIEL - RS
}

Bruna Nascimento de Vasconcellos ${ }^{1}$, Aline Bernarda Debastiani ${ }^{1}$, Mônica Joelma do Nascimento Anater ${ }^{2}$, Ana Paula Dalla Corte ${ }^{3}$, Carlos Roberto Sanquetta

1 Doutoranda em Engenharia Florestal, Universidade Federal do Paraná, Curitiba, PR, Brasil-brunasig@hotmail.com

2 Mestranda em Bioenergia, Universidade Federal do Paraná, Curitiba, PR, Brasil 3 Prof. Dr. do Departamento de Engenharia Florestal, Universidade Federal do

Paraná, Curitiba, PR - Brasil

Recebido em: 03/10/2016 - Aprovado em: 21/11/2016 - Publicado em: 05/12/2016 DOI: 10.18677/EnciBio_2016B_097

O presente estudo teve como objetivo analisar as mudanças do uso e ocupação da terra no município de São Gabriel - RS, e testar a acurácia de diferentes algoritmos classificadores. $O$ trabalho foi realizado nas seguintes etapas: processamento das imagens e georreferenciamento; realização da classificação supervisionada com os algoritmos distância mínima, paralelepípedo e máxima verossimilhança; análise das matrizes de confusão e dos erros de classificação, e realização da análise multitemporal com a melhor classificação. A classe campo foi a que apresentou maior abrangência, sendo de 40,48 \% para 2009 e de 33,54\% para 2015, o decréscimo está relacionado ao aumento das classes floresta e agricultura. Dentre os algoritmos testados, o que apresentou melhores resultados foi o de Máxima Verossimilhança.

PALAVRAS-CHAVE: Landsat, Máxima Verossimilhança, Sensoriamento Remoto.

\section{ALGORITHMS TEST CLASSIFICATION FOR TEMPORARY ANALYSIS OF USE AND LAND OCCUPATION IN THE MUNICIPALITY OF SÃO GABRIEL - RS}

\begin{abstract}
This study aims to analyze the land use and occupation changes in São Gabriel - RS, and test the accuracy of different classifiers algorithms. The work was performed in the following steps: image processing and georeferencing; realization of supervised classification algorithms with the minimum distance, parallelepiped and maximum likelihood; analysis of arrays of confusion and misclassification, and realization of multi-temporal analysis with the best rating. The field class was the one with greater coverage, and of $40.48 \%$ for 2009 and $33.54 \%$ for 2015 , the decrease is related to the increase in forest and agriculture classes. Among the tested algorithms, which showed better results was the Maximum Likelihood.
\end{abstract}

KEYWORDS: Landsat, maximum likelihood, Remote Sensing.

\section{INTRODUÇÃO}

A falta de planejamento e destruição dos recursos naturais, bem como a retirada de florestas e da vegetação nativa contribuem para redução do espaço dos ENCICLOPÉDIA BIOSFERA, Centro Científico Conhecer - Goiânia, v.13 n.24; p. 1034 2016 
ecossistemas naturais, contribuindo para o assoreamento de rios, o desequilíbrio da fauna e flora, e demais problemas ambientais. Diante do dinamismo das ações humanas, não é possível acompanhar tais mudanças sem o auxílio de tecnologias, de modo que, é necessário o uso de técnicas e produtos de sensoriamento remoto, hoje disponíveis gratuitamente, que possibilitam obter de forma rápida e segura o mapeamento de uso e ocupação da terra (LIPPERT et al., 2012).

Nos últimos anos tornou-se relevante analisar as mudanças de uso e cobertura da terra no intuito de compreender as dinâmicas e processos que atuam nos diferentes espaços, auxiliar no planejamento ambiental e definir áreas prioritárias para recuperações e investimentos (MARCHESAN et al., 2016). Dentre as técnicas utilizadas para o mapeamento de uso e ocupação da terra, tem-se a classificação de imagens, que se baseia na distinção e identificação de diferentes alvos que possuem comportamento espectral distinto, de modo que, associa-se cada pixel da imagem a um determinado rótulo, apresentando um tema como resultado.

A classificação supervisionada utiliza algoritmos pré-estabelecidos, onde o usuário identifica pontos nas imagens pertencentes às classes desejadas e o software executa a tarefa de localizar os demais pixels pertencentes àquelas classes, baseado em alguma regra estatística pré-estabelecida (CROSTA, 1992). No entanto, para obter bons resultados com um algoritmo supervisionado, muitas vezes é necessário coletar uma grande quantidade de dados de treinamento, principalmente ao que se refere a classes mais heterogêneas, permitindo a representação de toda a diversidade da classe (ROUGIER et al., 2016).

O mapeamento de cobertura da terra apresenta diversos fatores que influenciam a qualidade do produto final. Tais fatores estão relacionados ao tipo de imagem utilizada, algoritmo de classificação, recursos de entrada, dados de validação, técnicas de pré e pós processamento, dados auxiliares e tipos de classes utilizadas. A comunidade de Sensoriamento Remoto empreendeu esforços consideráveis para melhorar a acurácia de mapeamento de uso e ocupação da terra (KHATAMI et al., 2016).

$\mathrm{Na}$ análise de acurácia da classificação utilizam-se estatísticas que visam verificar se os alvos estão atribuídos corretamente nas classes. Por meio da matriz de confusão, é possível calcular os índices de avaliação, onde os valores das linhas e colunas correspondem aos números de pixels atribuídos a uma classe em relação a sua identificação correta, conforme análise da verdade de campo ou em mapa de referência (NOVO, 2010). Diante disso, o presente estudo objetivou o desenvolvimento de testes de diferentes algoritmos de classificação supervisionada para a realização da análise temporal de uso e ocupação da terra no município de São Gabriel - RS.

\section{MATERIAL E MÉTODOS}

\section{Caracterização da área de estudo}

O município de São Gabriel localiza-se na microrregião da Campanha Central do estado do Rio Grande do Sul, Brasil, conforme pode ser visualizado na Figura 1. A população estimada pelo Instituto Brasileiro de Geografia e Estatística (IBGE) é de aproximadamente 60 mil habitantes. 


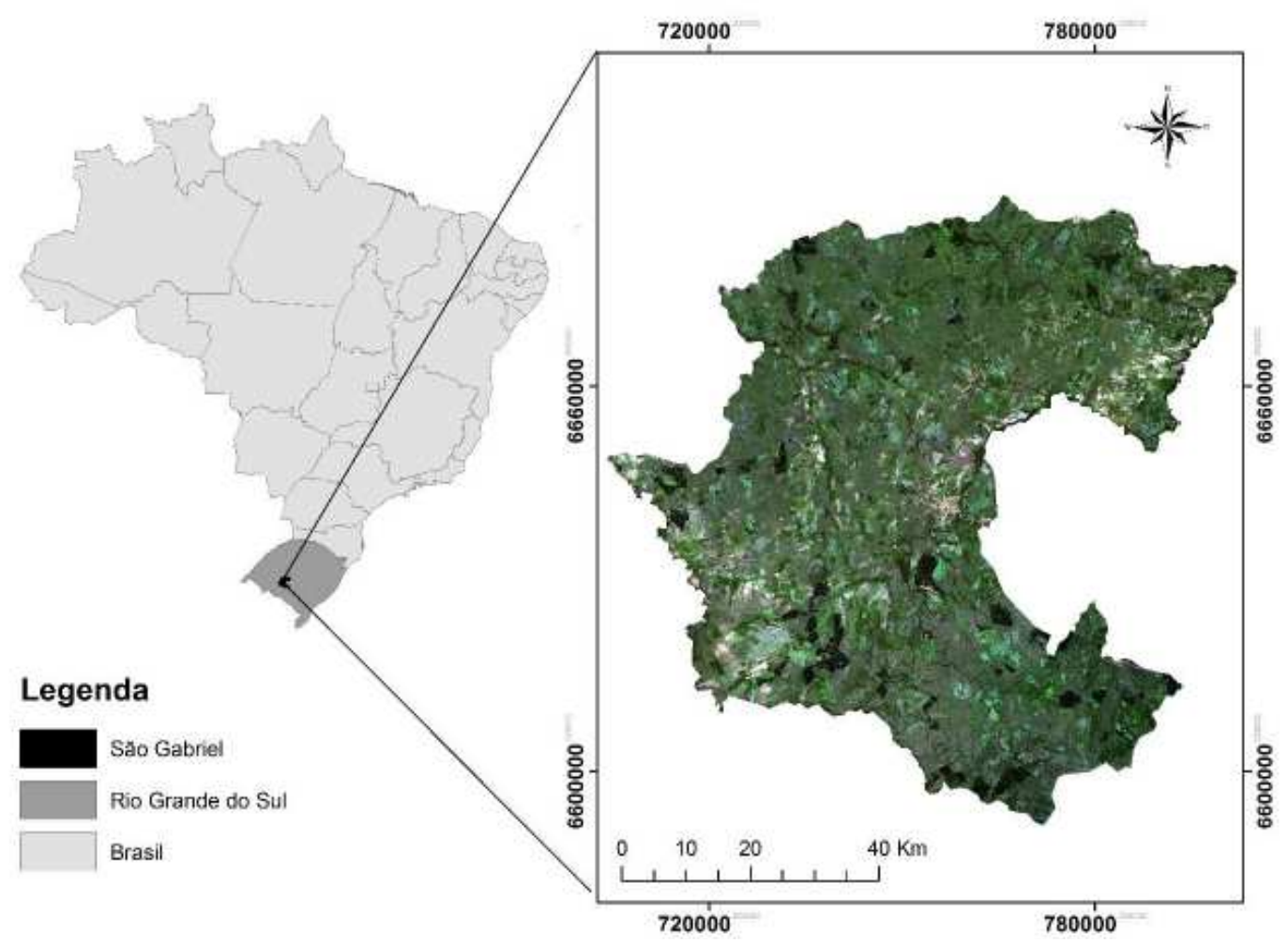

FIGURA 1 - Localização do município de São Gabriel - RS

\section{Materiais Utilizados}

- Imagens orbitais dos satélites Landsat 5 sensor TM (Thematic Mapper) referente a 30/06/2009 (bandas 1, 2, 3, 4, 5, 6 e 7) e do Landsat 8 sensor OLI/TIRS (Operational Land Imager e Thermal Infrared Sensor) referente a 16/06/2015 (bandas 2, 3, 4, 5, 6, 7 e 9), disponível na Divisão de Geração de Imagens (DGI) do Instituto Nacional de Pesquisas Espaciais (INPE).

- Carta topográfica do município de São Gabriel, obtida no banco de dados geográficos do Exército, disponibilizada pelo Geoportal - Infraestrutura Nacional de dados espaciais (Exército Brasileiro) na escala 1:100.000, de nomenclatura S-H-21-Z-B-III;

- Software Envi 5.0.

\section{Georreferenciamento}

O georreferenciamento consiste em conceder a uma imagem um sistema de coordenadas do mundo real e corrigir eventuais deformações decorrentes do processo de aquisição da imagem de satélite (ECKHARDT et al., 2013). O georreferenciamento foi realizado utilizando pontos de controle obtidos na carta topográfica de São Gabriel disponibilizada pelo Exército Brasileiro.

O erro quadrático máximo (RMS) admitido foi de 0,5 pixel. De acordo com MENESES \& ALMEIDA (2012), o valor de RMS é expresso em números de pixels. Por exemplo, um erro RMS de valor dois, significa que o pixel retransformado está distante dois pixels do pixel de referência. Se os pontos de controle mostram valores altos de RMS na direção $X$ ou na direção $Y$, deve-se adicionar mais pontos naquela direção. 


\section{Classificação supervisionada}

Foram utilizados três algoritmos classificadores, sendo: Mínima Distância, Paralelepípedo, e Máxima Verossimilhança. O método da mínima distância utiliza a média dos pixels da amostra, para assinalar um pixel desconhecido a uma determinada classe. O pixel é atribuído a classe que apresentar a menor distância, independente da banda (AMORIM et al., 2016).

De acordo com MENESES \& ALMEIDA (2012), o classificador paralelepípedo tem esse nome porque em um espaço de dados bidimensional (duas bandas), os valores dos pixels de cada classe de treinamento são estimados por um limite superior e um inferior de valores digitais, que são identificados como as dimensões das bordas do paralelepípedo. Para estimar esses limites tomam-se os valores terminais da curva dos histogramas das amostras de treinamento em cada banda. É frequentemente usado como uma primeira exploração de classificação, a fim de identificar quantas classes podem ser utilizadas nos demais tipos de classificação.

O método da máxima verossimilhança (MaxVer) é o mais comum, considera a ponderação das distâncias entre médias dos níveis digitais de classes, utilizando parâmetros estatísticos. Porém, para que o classificador apresente um bom desempenho é necessário um número elevado de pixels para cada conjunto de treinamento (IZIPPATO et al., 2012).

\section{Avaliação da classificação}

De acordo com NOVO (2010), após a classificação, torna-se necessário a avaliação da exatidão dessa classificação, ou seja, é necessário determinar o quão bom é o resultado da classificação em relação a realidade. Para analisar a qualidade da classificação foi utilizada a Exatidão Global (G), Coeficiente Kappa (K) e o Tau $(T)$, que podem ser visualizados nas Equações 1, 2 e 3.

$$
\begin{gathered}
G=\frac{\sum_{i=1}^{M} n_{i i}}{N}=P_{0} \\
K=\frac{P_{0}-P_{e}}{1-P_{e}} \\
T=\frac{P_{0}-P_{r}}{1-P_{r}}
\end{gathered}
$$

Onde: $n_{i i}=$ Número de pixels da diagonal principal da Matriz de Confusão de cada classe; $N=$ Número de classes; $P_{0}=$ Concordância total ou Exatidão global; $P_{r}$ : concordância ao acaso esperada; $P_{r}$ : concordância esperada.

Para cada matriz de erro pode ser calculado o coeficiente Kappa, sendo a medida da concordância da classificação com os dados de referência, o valor desse índice pode variar de 0 a 1 , de modo que 0 significa péssima classificação e 1 significa excelente, conforme pode ser visualizado na Tabela 1. 
TABELA 1 - Índice kappa e sua concordância

\begin{tabular}{c|c}
\hline Índice Kappa & Concordância \\
\hline 0,00 & Péssima \\
0,01 a 0,20 & Ruim \\
0,21 a 0,40 & Razoável \\
0,41 a 0,60 & Boa \\
0,61 a 0,80 & Muito Boa \\
0,81 a 1,00 & Excelente \\
\hline
\end{tabular}

Fonte: LANDIS \& KOCH, (1977).

\section{RESULTADOS E DISCUSSÃO}

A análise temporal permitiu identificar as mudanças ocorridas no período entre 2009 e 2015, estas podem ser visualizadas na Tabela 2 e Figura 2.

TABELA 2 - Área de cada classe de uso e ocupação da terra

\begin{tabular}{ccccc}
\hline \multirow{2}{*}{ Classe } & \multicolumn{2}{c}{ Ano de 2009 } & \multicolumn{2}{c}{ Ano de 2015 } \\
\cline { 1 - 2 } & Área (ha) & Área (\%) & Área (ha) & Área (\%) \\
\cline { 1 - 4 } Recursos hídricos & 234,60 & 4,67 & 236,20 & 4,70 \\
Campo & 2034,12 & 40,48 & 1685,20 & 33,54 \\
Agricultura & 625,40 & 12,45 & 516,73 & 10,28 \\
Floresta plantada & 687,20 & 13,68 & 924,12 & 18,39 \\
Floresta nativa & 656,10 & 13,06 & 964,28 & 19,19 \\
Solo exposto & 787,50 & 15,67 & 698,20 & 13,90 \\
\hline
\end{tabular}

A área aproximada do município de São Gabriel é de aproximadamente 5024,90 hectares. A classe de uso e ocupação da terra que apresenta a maior abrangência nos anos de 2009 e 2015 é a de campo, com 40,48\% e 33,54\% respectivamente. Este predomínio de campo já era esperado na área de estudo, visto que é característico do bioma pampa, que apresenta uma paisagem desprovida de elementos arbóreos e arbustivos, sendo composto por gramíneas. 

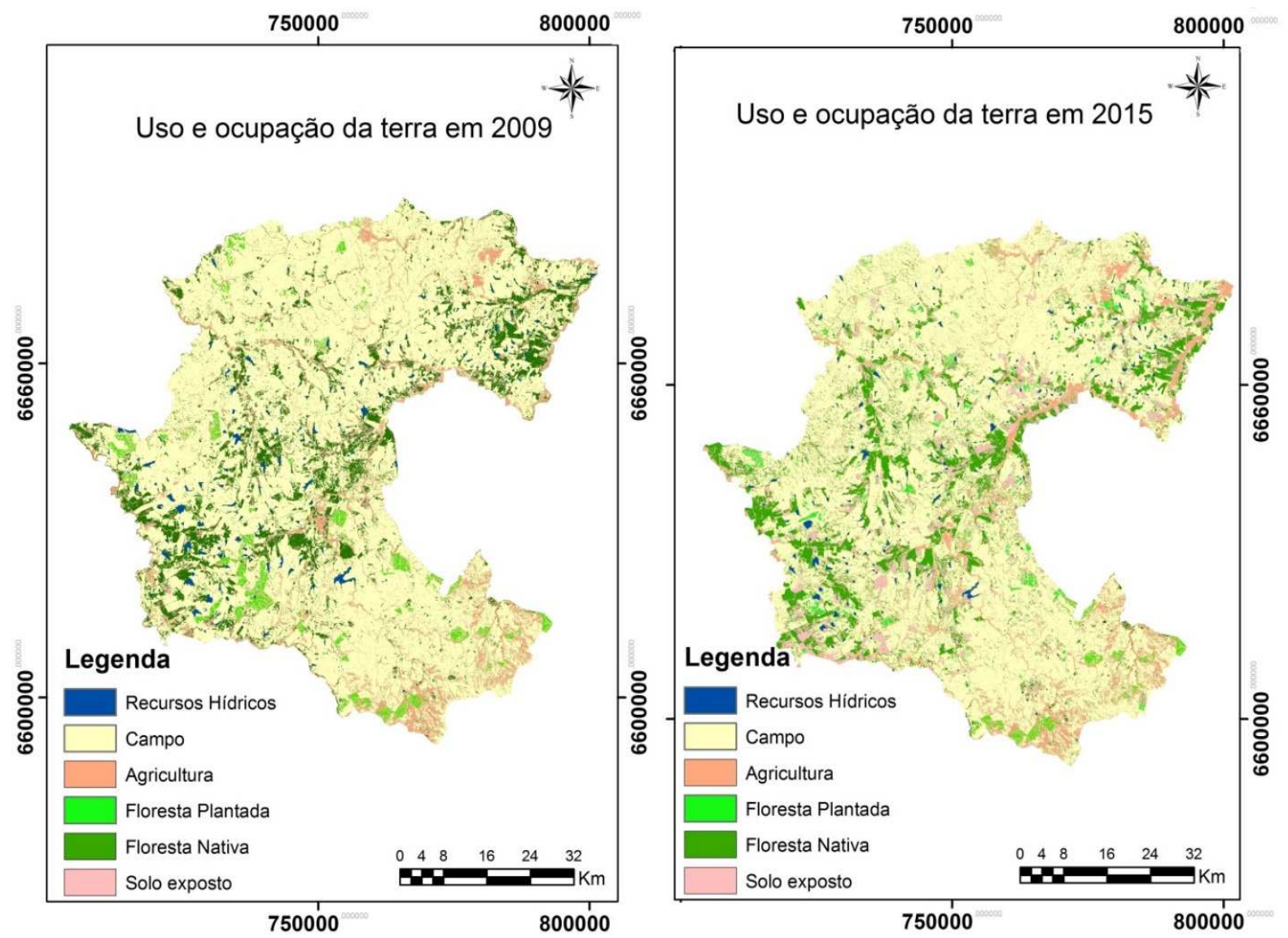

FIGURA 2 - Distribuição espacial das classes de uso e ocupação da terra nos anos de 2009 e 2015.

Entre 2009 e 2015 houve um aumento na cobertura florestal no município de São Gabriel, sendo de $4,71 \%$ de florestas plantadas e de $6,13 \%$ de floresta nativa. De acordo com BENEDETTI et al. (2013), o Rio Grande do Sul apresentou uma expansão florestal a partir do ano de 2003, onde o governo lançou o Programa Estadual de Florestamento, que previa fomentar o plantio comercial em municípios da metade sul do estado. Além do que, a expansão florestal é dada de duas maneiras, podendo ser relacionada ao estabelecimento da regeneração e posterior formação florestal nativa, e ainda, por meio da introdução de espécies exóticas como Pinus e Eucalyptus.

CRASHAW et al. (2007) consideram que a silvicultura é potencialmente ameaçadora às áreas de campo no Rio Grande do Sul, pois um dos programas de fomento estadual apresentava como meta o plantio de 10 milhões de hectares. A classe campo teve um decréscimo de $6,94 \%$ no período, o que pode estar diretamente relacionado ao investimento florestal no estado e ao aumento da agricultura, que foi de 2,17\%. De acordo com SANTOS \& SILVA (2011), nas últimas décadas, várias áreas cobertas com campos foram transformadas para outros usos, bem como em pastagens, cultivos intensivos e silvicultura, além da invasão de espécies invasoras ou introduzidas pelos produtores. CRASHAW et al. (2007) consideram que o Rio Grande do Sul teve desenvolvimento econômico primordial vinculado as atividades pastoris em função das pastagens naturais, que são a base dos rebanhos bovino, ovino e equino. 
SANTOS \& SILVA (2011) utilizaram imagens do satélite Landsat para identificar as modificações na paisagem e problemas do bioma pampa. O estado de conservação do bioma pampa é pouco conhecido, pois a cobertura dos remanescentes é pouco documentada, de modo que, é necessário o monitoramento e investimento na recuperação e recomposição de áreas degradadas em regiões chaves para incrementar o grau de conectividade da paisagem nesse bioma. $\mathrm{Na}$ Tabela 3 podem ser visualizados os resultados dos índices utilizados para avaliação da classificação.

TABELA 3 - Valores de exatidão global, kappa e tau para as classificações testadas.

\begin{tabular}{cccccc}
\hline Sensor & Classificação & $\mathbf{G}(\%)$ & $\mathbf{K}$ & $\mathbf{T}$ & Ranking \\
\hline \multirow{3}{*}{ Landsat 5 } & Maxver & 97,35 & 0,9687 & 0,996 & 1 \\
& Distância mínima & 91,43 & 0,8987 & 0,885 & 2 \\
& Paralelepípedo & 84,39 & 0,8143 & 0,867 & 3 \\
\hline \multirow{2}{*}{ Landsat 8 } & Maxver & 99,70 & 0,9964 & 0,964 & 1 \\
& Distância mínima & 94,05 & 0,9278 & 0,920 & 2 \\
& Paralelepípedo & 84,30 & 0,8364 & 0,839 & 3 \\
\hline
\end{tabular}

Onde: G: Exatidão Global; K: Índice Kappa; T: Índice Tau

De acordo com a classificação proposta por LANDIS \& KOCH (1977) o valor do índice kappa para todos os classificadores é enquadrada como tendo a sua concordância excelente, de modo que, a partir de 0,80 a classificação se aproxima muito da "verdade terrestre". Os indicadores de exatidão global e tau também apresentaram resultados satisfatórios. Quanto a acurácia dos classificadores considerando a exatidão global, Kappa e Tau, os melhores resultados foram gerados pelo algoritmo MaxVer, seguido por distância mínima e paralelepípedo.

NASCIMENTO et al. (2016) avaliaram a exatidão dos classificadores MaxVer e Isso cluster do software ArcGis com uso de imagens Landsat. A exatidão global, TAU e coeficiente Kappa foram de 52\%, 31\% e 44\% para o algoritmo MaxVer e 53\%, $29 \%$ e $45 \%$ respectivamente. Ambos os classificadores tiveram um desempenho moderado, porém, o MaxVer ainda apresentou os melhores resultados. MELLO et al. (2012) avaliaram técnicas de classificação supervisionada de imagens Landsat para detecção da cobertura da terra em Rondônia. Dentre os classificadores, o melhor resultado foi gerado por MaxVer, seguido por Paralelepípedo e Mahalanobis.

\section{CONCLUSÃO}

Por meio das análises realizadas, foi possível perceber que o algoritmo da máxima verossimilhança foi o que apresentou os melhores resultados. $A$ análise temporal permitiu identificar a variação nas classes temáticas do período, sendo considerada uma ferramenta útil no mapeamento e monitoramento da vegetação. 


\section{REFERÊNCIAS}

AMORIM, R.M.; ALBARICI, F.L.; PINO, M.A.I.T. Mapa digital do escoamento superficial por meio de imagens de sensor remoto na sub-bacia do Rio Mogi Guaçu - MG. Revista Brasileira de Geografia Física, v.9, n.3, p.1-17, 2016. Disponível em: http://www.revista.ufpe.br/rbgfe/index.php/revista/article/view/1604/968

BENEDETTI, A.C.P.; LIPPERT, D.B.; PEREIRA, R.S.; ALMEIDA, C.M.; CARDOSO, C.D.V.; HENDGES, E.R. Uso do produto MOD13Q1 do sensor MODIS para análise temporal e mapeamento das florestas nas serras do sudeste e campanha meridional do Rio Grande do Sul. Revista Árvore, v.37, n.3, p. 459-469, 2013. Disponível em: http://dx.doi.org/10.1590/S0100-67622013000300009

CRASHAW, D.; DALL'AGNOL, M.; CORDEIRO, J.L.P.; HASENACK, H. Caracterização dos campos sul-riograndenses: uma perspectiva da ecologia da paisagem. Boletim gaúcho de geografia, v. 33, p.233-252, 2007. Disponível em: http://seer.ufrgs.br/index.php/bgg/article/view/37437/24182

CROSTA, A.P. Processamento digital de imagens de sensoriamento remoto. Campinas: IG/UNICAMP, 1992, 170 p.

ECKHARDT, R.R.; SILVEIRA, C.A.; REMPEL, C. Evolução temporal do uso e cobertura da terra no município de Bom Retiro do Sul- RS, Brasil. Caminhos de geografia, v.14, n.47, p.150-161, 2013. Disponível em: http://seer.ufrgs.br/index.php/bgg/article/view/37437/24182

IZIPPATO, F.J.; MIRANDOLA, P.H.; SILVA, R.A.; PIRES, E.V.R. Análise ambiental no sistema de Bacia Hidrográfica do córrego Rio Branco com fins de planejamento ambiental com uso de geotecnologias (1985/2011) - Três Lagoas/MS. Revista Geonorte, v.2, n.4, p.1180-1194, 2012. Disponível em: http://www.periodicos.ufam.edu.br/index.php/revista-

geonorte/article/viewFile/2180/2050

KHATAMI, R.; MOUNTRAKIS, G.; STEHMAN, S.V. A meta-analysis of remote sensing research on supervised pixel-base land cover image classification processes: General guidelines for practitioners and future research. Remote Sensing of Enviroment, v.177, p. 89-100, 2016. Disponível em: http://dx.doi.org/10.1016/j.rse.2016.02.028

LANDIS, J.; KOCH, G. The measurement of observer agreement for categorical data, Washington, USA. Biometrics, v.33, n.1, p.159-174, 1977. Disponível em: https://www.jstor.org/stable/2529310?seq=1\#page_scan_tab_contents

LIPPERT, D.B.; BENEDETTI, A.C.P.; PEREIRA, R.S.; Dinâmica da cobertura florestal no município de Crissiumal - RS durante o período de vinte anos. Revista Eletrônica em Gestão, Educação e Tecnologia, v.7, n.7, p.1297-1305, 2012. Disponível em: http://dx.doi.org/10.5902/223611705543

MARCHESAN, J.; PEDRALI, L.D.; TRAMONTINA, J.; ALBA, E.; MELLO, E.P.; PEREIRA, R.S. Análise da relação entre a evolução florestal e a declividade 
utilizando imagens Landsat. Nativa, v.4, n.1; p. 53-57, 2016. DOI: 10.14583/23187670.v04n01a11

MELLO, A. Y. I.; ALVES, D. S. A.; LINHARES, C. A.; LIMA, F. B. L. Avaliação de técnicas de classificação digital de imagens Landsat em diferentes padrões de cobertura da terra em Rondônia. Revista Árvore, v.36, n.3, p.537-547, 2012. Disponível em: http://www.redalyc.org/articulo.oa?id=48822958016

MENESES, P.R.; ALMEIDA, T.; Introdução ao processamento de imagens de sensoriamento remoto. Unb, Brasília, 2012, 266p.

NASCIMENTO, I.S.; CRUZ, C.B.M.; NEVES, S.M.A.S.; GALVANIN, E.A.S. Avaliação da exatidão dos classificadores Maxver e Isso cluster do software Arcgis for desktop. Revista continentes, ano 5, n.8, p.48-62, 2016. Disponível em: http://r1.ufrrj.br/revistaconti/index.php/continentes/article/view/118

NOVO, E.M.L.M.; Sensoriamento Remoto: princípios e aplicações. 4. Ed. São Paulo - SP, Blucher, 2010.

ROUGIER, S.; PUISSANT, A.; STUMPF, A.; LACHICHE, N.; Comparison of sampling strategies for object-based classification of urban vegetation from Very High Resolution satellite images. International Journal of Applied Earth Observation and Geoinformation, v.51, p.60-73, 2016. Disponível em: http://dx.doi.org/10.1016/j.jag.2016.04.005

SANTOS, S.; SILVA, L.G.; Mapeamento por imagens de sensoriamento remoto evidencia o bioma pampa Brasileiro sob ameaça. Boletim de Geografia, v.29, n.2, p.49-57, 2011. Disponível em: http://dx.doi.org/10.4025/bolgeogr.v29i2.12366 